\title{
Carcinoma del uraco: reporte de un caso y revisión de la bibliografía
}

\author{
Carcinoma of the urachus: A case report and literature \\ review
}

Jorge Gerardo Sandoval-Téllez, ${ }^{1}$ Abel A. Ricardez-Espinosa, ${ }^{2}$ Miguel J. Suárez-Uriarte, ${ }^{1}$ Itzel Reyes-De la Garza, ${ }^{3}$ Luis A. Jiménez-López ${ }^{4}$

\begin{abstract}
Resumen
ANTECEDENTES: Los tumores del uraco constituyen menos de $1 \%$ de las neoplasias de vejiga y aproximadamente 20 a $35 \%$ de los adenocarcinomas vesicales. Hasta la fecha se sabe poco de esta patología; la etiología y patogénesis son aún inciertas y no se han identificado factores de riesgo para padecer estos tumores.

CASO CLínICO: Paciente masculino de 41 años de edad, quien acudió al servicio de Urología por hematuria macroscópica, no formadora de coágulos, asociada con dolor en el hipogastrio. A la exploración física se observó el abdomen con discreto aumento de volumen en el hipogastrio y a la palpación un tumor de aproximadamente $5 \mathrm{~cm}$, dependiente de la cicatriz umbilical, móvil, no doloroso ni fijo en los planos profundos, ligeramente indurado. La tomografía abdominopélvica mostró la vejiga urinaria con engrosamiento de la pared y en la línea media una imagen irregular, heterogénea, de $38 \times 27$ mm, con múltiples calcificaciones periféricas puntiformes. La cistoscopia evidenció un tumor en la cúpula vesical de aspecto sólido, con zonas de color marrón, diámetro aproximado de $3 \mathrm{~cm}$ y bordes irregulares. El tratamiento consistió en cistectomía parcial y resección en bloque del uraco, además de linfadenectomía y omentectomía, con resultados satisfactorios.

CONCLUSIONES: El carcinoma del uraco es una alteración poco frecuente; la mayor parte de la información proviene de reportes y series de casos. El tratamiento de elección consiste en cistectomía parcial y resección en bloque del uraco, con la que se obtienen buenos resultados, enfermedad libre de progresión y supervivencia de 40 a $60 \%$, según el tipo de procedimiento quirúrgico implementado.
\end{abstract}

PALABRAS CLAVE: Carcinoma; uraco; cistectomía.

Abstract

BACKGROUND: Tumors of the urachus make up less than $1 \%$ of the bladder neoplasia and approximately 20 to $35 \%$ of bladder adenocarcinomas. At present, little is known of the pathology. Etiology and pathogenesis are still uncertain and risk factors for those tumors have not been identified.

CLINICAL CASE: A 41-year-old man came to the urology service due to gross hematuria with no clot formation, accompanied by pain in the hypogastrium. Physical examination revealed an abdomen with a slightly increased volume in the hypogastrium. A mobile, nonpainful, mildly indurated tumor dependent on the umbilicus, measuring approximately $5 \mathrm{~cm}$ and not fixed to the deep planes was palpated. Abdominopelvic tomography identified a urinary bladder with wall thickening and a heterogeneous, irregular image measuring $38 \times 27 \mathrm{~mm}$ at the midline, with punctiform peripheral calcifications. Cystoscopy showed a solid tumor in the bladder dome with a diameter of $3 \mathrm{~cm}$, brown areas, and irregular edges. Treatment was partial cystectomy and in bloc resection of the urachus, in addition to lymphadenectomy and omentectomy.

CONCLUSIONS: Carcinoma of the urachus is a rare alteration and most information on it comes from case reports and case series. The treatment of choice is partial cystectomy and en bloc resection of the urachus, which has provided good results and progressionfree survival rates from 40 to $60 \%$, depending on the type of procedure performed.

KEYWORDS: Carcinoma; Urachus; Cystectomy.
${ }^{1}$ Residente de Urología.

${ }^{2}$ Adscrito al servicio de Urología.

${ }^{3}$ Departamento de Anatomía Patológica. ${ }^{4}$ Jefe del servicio de Urología.

Hospital de Especialidades 14, UMAE Centro Médico Nacional Lic. Adolfo Ruiz Cortines, Instituto Mexicano del Seguro Social, Veracruz, México.

Recibido: noviembre 2017

Aceptado: mayo 2018

Correspondencia

Jorge Gerardo Sandoval Téllez drgerardosandoval@gmail.com

Este artículo debe citarse como Sandoval-Tellez JG, Ricardez-Espinosa AA, Suarez-Uriarte MJ, Reyes-De La Garza I, Jiménez-López A. Carcinoma del uraco: reporte de un caso y revisión de la bibliografía. Rev Mex Urol. 2018 mayo-junio;78(3):215-219.

DOI:https://doi.org/10.24245/revmexurol.v78i3.1808 


\section{ANTECEDENTES}

Los tumores del uraco constituyen menos de 1\% de las neoplasias de vejiga y aproximadamente 20 a 35\% de los adenocarcinomas vesicales. ${ }^{1} \mathrm{El}$ uraco es un remanente embriológico del alantoides, identificado como una estructura o cordón fibroso desde el ombligo hasta la cúpula vesical, que suele obstruirse a partir del nacimiento. ${ }^{2}$ Hasta la fecha se sabe poco del carcinoma: la etiología y patogénesis son aún inciertas y no se han identificado factores de riesgo. ${ }^{3}$ El tratamiento de elección consiste en cistectomía parcial, con exéresis completa del tumor. ${ }^{4,5}$

\section{CASO CLÍNICO}

Paciente masculino de 41 años de edad, quien acudió al servicio de Urología del Centro Médico Nacional Lic. Adolfo Ruiz Cortines (IMSS) por hematuria macroscópica, no formadora de coágulos, asociada con dolor en el hipogastrio. Refirió pérdida ponderal de $10 \mathrm{~kg}$ en tres meses. A la exploración física se observó el abdomen con discreto aumento de volumen en el hipogastrio y a la palpación un tumor de aproximadamente $5 \mathrm{~cm}$, dependiente de la cicatriz umbilical, móvil, no doloroso ni fijo en los planos profundos, ligeramente indurado.

Los estudios de laboratorio reportaron citometría hemática dentro de los parámetros normales; química sanguínea de 3 elementos en ayuno de $8 \mathrm{~h}$ con glucemia de $164 \mathrm{mg} / \mathrm{dL}$, urea de $40 \mathrm{mg} /$ $\mathrm{dL}$ y creatinina de $1.1 \mathrm{mg} / \mathrm{dL}$. El examen general de orina con glucosuria, proteinuria, hemoglobina (+) y numerosos eritrocitos. El ultrasonido abdominal de vejiga evidenció una imagen lobulada, de contenido heterogéneo adyacente a la pared superior. La tomografía abdominopélvica (Figuras 1 y 2) reportó: retroperitoneo libre de adenopatías, morfología de grandes vasos con diámetro y trayecto normales; en la cavidad pélvica se observó la vejiga urinaria con engro-

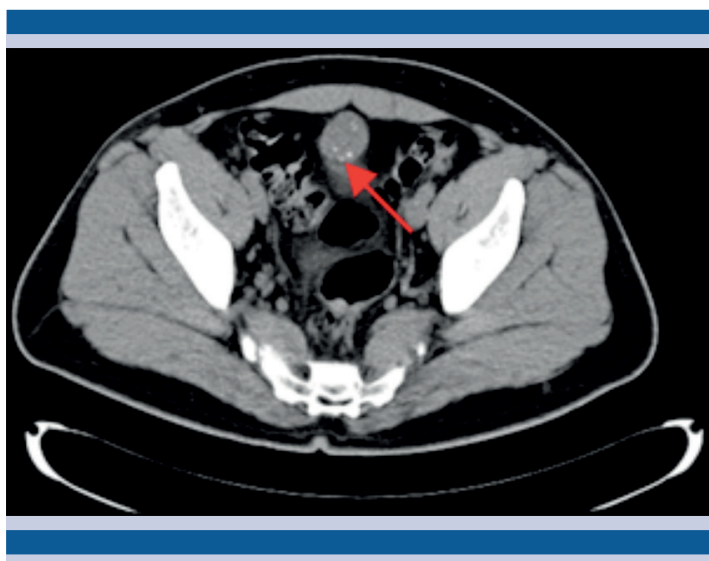

Figura 1. Tomografía (corte axial) que muestra múltiples calcificaciones periféricas puntiformes.

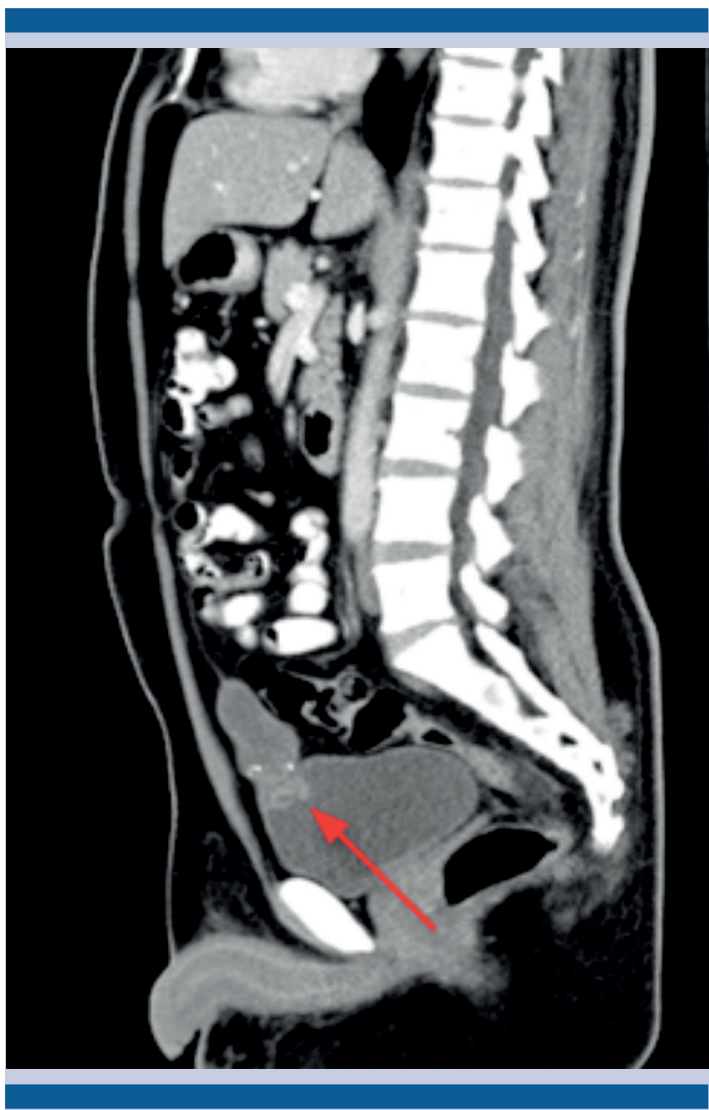

Figura 2. Tomografía contrastada (corte coronal) que evidencia el tumor, con infiltración en el domo vesical.

samiento de la pared y en la línea media la pared anterior con imagen irregular, heterogénea, que 
se realzaba con el medio de contraste, con extensión hacia la cavidad y pared abdominales, de 38 × 27 mm, con múltiples calcificaciones periféricas puntiformes.

La cistoscopia (Figura 3) evidenció un tumor en la cúpula vesical de aspecto sólido, con zonas de color marrón, diámetro aproximado de $3 \mathrm{~cm}$ y bordes irregulares. El tratamiento consistió en resección transuretral de la vejiga, con resultados satisfactorios.

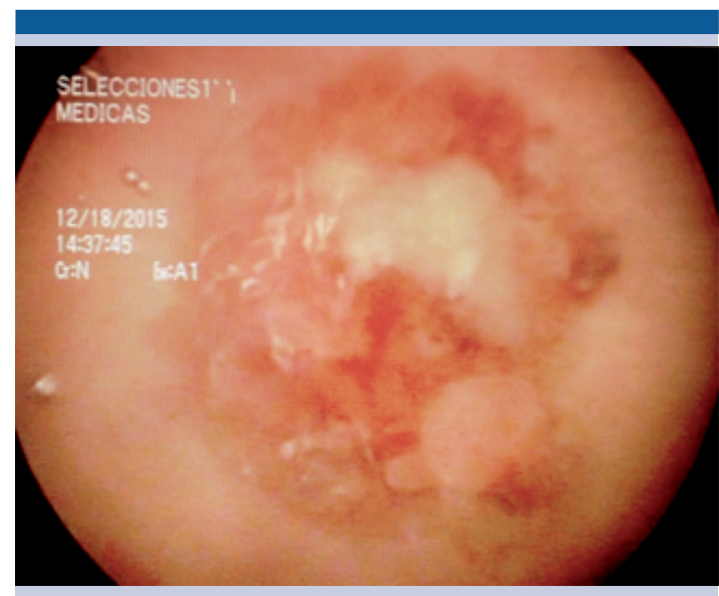

Figura 3. Tumor vesical.

\section{Hallazgos histopatológicos}

El tejido evaluado se procesó con la técnica histológica convencional (se realizaron secciones de 3 micras para teñirse con hematoxilina y eosina). Se identificó una neoplasia con grupos de luces glandulares, epitelio mucosecretor con núcleos polarizados en la base, citoplasma claro, coexistencia de mucina y áreas con epitelio de tipo transicional, que infiltraban el tejido adiposo adyacente, asociado con reacción desmoplásica (inflamación y fibrosis peritumoral) (Figura 4). La muestra se envió a marcaje con anticuerpos de citoqueratina 7 , que resultó positiva-membranosa $(+++)$, citoqueratina $20(+++)$, CDX2



Figura 4. Citología que muestra grupos de luces glandulares, epitelio mucosecretor con núcleos polarizados en la base y citoplasma claro.

positivo-nuclear $(+++)$ y antígeno prostático específico negativo. Este inmunofenotipo se expresa en tumores glandulares de origen uracal, con patrón de crecimiento glandular, por lo que la positividad de citoqueratina 7 descarta la metástasis gastrointestinal y la negatividad para el antígeno prostático descarta la posibilidad de metástasis de origen prostático.

Se realizó tratamiento quirúrgico abierto, accediendo por la línea media, donde se encontró carcinomatosis peritoneal. Se llevó a cabo cistectomía parcial y resección en bloque del uraco, además de linfadenectomía y omentectomía. El reporte de histopatología fue: adenocarcinoma mucoproductor con escasas glándulas tubulares, moderadamente diferenciado, que afectaba la mucosa y la pared hasta la capa muscular de la vejiga, además de dos implantes tumorales en el omento de 3 y $5 \mathrm{~cm}$. Los ganglios linfáticos mostraron, solamente, hiperplasia sinusoidal reactiva.

El paciente recibió quimioterapia coadyuvante con cisplatino y gemcitabina. Después de 10 meses de tratamiento persistió la enfermedad, con actividad ganglionar en la parte izquierda del 
cuello, axila ipsilateral, al igual que en la zona paraaórtica y hepática, por lo que se sustituyó el esquema a paclitaxel y carboplatino.

Los efectos adversos provocados por la quimioterapia fueron poco tolerados por el paciente; por tanto, decidió suspender el tratamiento después de un mes de haberlo iniciado. Actualmente recibe quimioterapia con capecitabina con fines paliativos y tramadol-paracetamol para controlar el dolor.

\section{DISCUSIÓN}

El carcinoma del uraco es un tumor excepcional, descrito por primera vez en 1931 por Begg. ${ }^{1}$ Comprende $0.17-0.34 \%$ de las neoplasias de vejiga y su incidencia anual se estima en 1 de cada 5 millones de pacientes. ${ }^{4}$ Afecta con mayor frecuencia a los hombres (1.8:1) de entre 50 y 60 años de edad. ${ }^{5}$ Hasta la fecha no se han identificado factores de riesgo para padecer este tipo tumores, de igual manera se ha descartado que la persistencia de la permeabilidad del uraco represente un factor predisponente para la expresión del carcinoma.

Existen menos de 400 casos reportados en la bibliografía desde su primera descripción (1931). ${ }^{5}$ Debido a su baja incidencia no es una enfermedad contemplada en las guías de práctica clínica estadounidenses ni europeas, por lo que el diagnóstico y tratamiento son poco claros.

Los adenocarcinomas constituyen el tipo histológico más frecuente de los tumores del uraco $(90 \%)$ y de estos, $63.6 \%$ son productores de mucina, $4.5 \%$ contienen células transicionales y $2.2 \%$ patrón mixto microcítico, de células transicionales y adenocarcinoma. El $10 \%$ restante de los tumores del uraco son uroteliales $(4 \%)$, sarcomatosos $(4 \%)$ e indiferenciados $(2 \%){ }^{6}$

Las manifestaciones clínicas pasan inadvertidas y suelen derivarse de la invasión del tumor a las estructuras vecinas; por tanto, con frecuencia se diagnostican en estadio avanzado. La hematuria (microscópica o macroscópica) es el síntoma predominante, incluso en $85 \%$ de los casos. También suele percibirse dolor en la región periumbilical (8.5\%), secreción fétida o sanguinolenta por el ombligo y secreción mucosa por la uretra (17\%); en algunos casos puede palparse la tumoración $(17 \%){ }^{7}$

El diagnóstico inicial se establece con estudios de imagen, como tomografía axial computada, resonancia magnética y ultrasonido. El hallazgo tomográfico más frecuente es la identificación de calcificaciones intravesicales $(70 \%) .^{8}$ En la cistoscopia se identifica un tumor localizado en la cúpula vesical o en la pared anterior $(91.5 \%)$; macroscópicamente tiene aspecto polipoide o ulcerado. La citología urinaria es positiva en $38 \%$ de los casos. ${ }^{7}$

Algunos estudios han reportado elevada concentración del antígeno carcinoembrionario, Ca 19-9 y Ca-125, principalmente cuando el origen de estos tumores es entérico; sin embargo, no son útiles para establecer el diagnóstico o seguimiento. ${ }^{9}$ La inmunohistoquímica expresa los marcadores para citoqueratina 20 (CK20) y el gen CDX-2 en $100 \%$ de los tumores del uraco y la citoqueratina 7 hasta en $50 \% .^{10}$

El tratamiento de elección consiste en cistectomía parcial con resección completa del uraco y el ombligo en bloque. La supervivencia de pacientes con cistectomía parcial es semejante a la de sujetos con cirugía radical. El procedimiento quirúrgico debe enfocarse en la preservación de la vejiga. La superviviencia a cinco años varía de 40 a $60 \% .^{5}$

Los factores de mal pronóstico incluyen: coexistencia de adenopatías, metástasis a distancia, márgenes quirúrgicos positivos, tamaño tumoral, diferenciación de células del anillo de sello, 
producción de mucina, afectación peritoneal, umbilectomía, linfadenectomía y cistectomía parcial versus radical (estas últimas no muestran diferencias significativas en cuanto a supervivencia respecta). ${ }^{2}$

El tratamiento coadyuvante con quimioterapia es imprescindible en todos los casos con ganglios regionales positivos. ${ }^{11}$ Los protocolos de quimioterapia combinada con cisplatino (metotrexato, vinblastina, doxorrubicina y cisplatino o gemcitabina-cisplatino) representan la primera línea en pacientes con carcinoma urotelial de vejiga y del uraco. El carcinoma del uraco comparte similitud histológica y clínica con el adenocarcinoma de colon; por tanto, la quimioterapia con 5-fluorouracilo también puede indicarse en pacientes con esta neoplasia. Se han publicado resultados prometedores con la combinación de 5-fluorouracilo, leucovorina, gemcitabina y cisplatino. ${ }^{2}$

Hasta la fecha no existe evidencia científica que respalde la indicación de radioterapia o quimioterapia como la mejor opción de tratamiento adyuvante. ${ }^{7}$ Los estudios que evalúan la quimioterapia coadyuvante han incluido pocos pacientes, establecido esquemas terapéuticos variables y no han aportado ningún nivel de evidencia. $^{3}$

\section{CONCLUSIONES}

El carcinoma del uraco es una alteración poco frecuente; la mayor parte de la información proviene de reportes y series de casos. El tratamiento de elección consiste en cistectomía parcial y resección en bloque del uraco, con la que se obtienen buenos resultados, enfermedad libre de progresión y supervivencia de 40 a $60 \%$, según el tipo de procedimiento quirúrgico implementado. Los factores de mal pronóstico incluyen metástasis a distancia y coexistencia de ganglios linfáticos. Existe poca evidencia relacionada con los esquemas de quimioterapia y su repercusión en la supervivencia global.

\section{REFERENCIAS}

1. Begg RC. The urachus: Its anatomy, histology and development. J Anat. 1930;64:170-83.

2. Szarvas T, Módos O, Niedworok $\mathrm{C}$, Reis $\mathrm{H}$, et al. Clinical, prognostic, and therapeutic aspects of urachal carcinoma-A comprehensive review with meta-analysis of 1,010 cases. Urol Oncol 2016;34(9):388-398.

3. Cruz P, Ramírez M, Iborra I, Rubio J, et al. Adenocarcinoma de uraco: ¿qué tanto sabemos? Rev Mex Urol 2014;74(5):301-307.

4. Sheldon CA, Clayman RV, González R, Williams R, Fraley EF. Malignant urachal lesions. J Urol 1984;131:1-8.

5. Herr HW, Bochner BH, Sharp D, Dalbagni G, Reuter VE. Urachal carcinoma: contemporary surgical outcomes. J Urol 2007;178:74-78.

6. Molina JR, Quevedo JF, Furth AF, Richardson RL, et al. Predictors of survival from urachal cancer. A Mayo Clinic study of 49 cases. Cancer 2007;110:2434-40.

7. Ashley RA, Inman BA, Sebo TJ, et al. Urachal carcinoma: Clinicopathologic features and long term outcomes of an aggressive malignancy. Cancer 2006;107:712-20.

8. Thali-Schwab CM, Woodward PJ, Wagner BJ. Computed tomographic appearance of urachal adenocarcinomas: Review of 25 cases. Eur Radiol 2005;15:79.

9. Siefker-Radtke AO, Gee J, Shen Y, et al. Multimodality management of urachal carcinoma: The M.D. Anderson experience. J Urol 2003;169:1295-8.

10. Paner GP, McKenney JK, Barkan GA, et al. Immunohistochemical analysis in a morphologic spectrum of urachal epithelial neoplasms: Diagnostic implications and pitfalls. Am J Surg Pathol 2011;35:787-98.

11. Meeks JJ, Herr HW, Bernstein M, et al. Preoperative accuracy of diagnostic evaluation of the urachal mass. J Urol 2013;189:1260-2. 